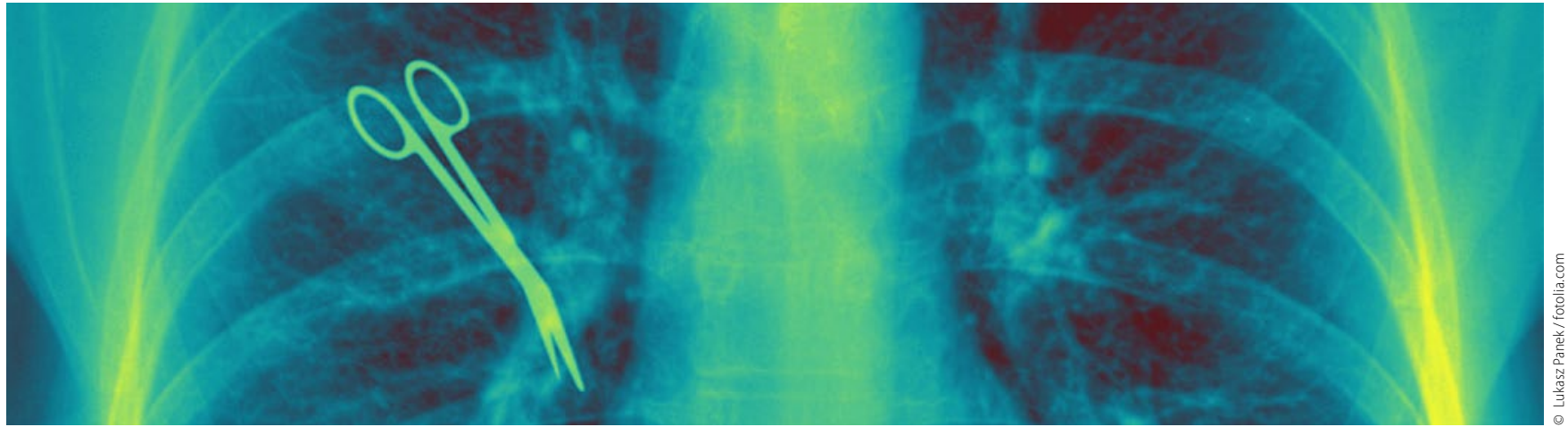

Neues und letztes Buch von Prof. Dr. Fritz Beske

\title{
„Die Schere zwischen Versorgungsbedarf und Ressourcen öffnet sich weiter"
}

\author{
Der demografische Wandel ist nicht mehr aufzuhalten - und in den Köpfen der Menschen ei- \\ gentlich auch präsent. „Aber die entscheidenden Punkte, die die Folgen der Demografie für das \\ Gesundheitswesen aufzeigen, werden von der Politik immer noch nicht bedacht." Mit dieser Ge- \\ neralkritik hat der renommierte Gesundheitssystem-Forscher Professor Dr. Fritz Beske Ende 2015 \\ sein neuestes Buch „Perspektiven des Gesundheitswesens - Geregelte Gesundheitsversorgung \\ im Rahmen der sozialen Marktwirtschaft" vorgestellt.
}

Es sei sein letztes Buch, kündigte der mittlerweile 93-Jährige an. „Und es ist das brisanteste Buch, das ich je geschrieben habe.“ Warum? Weil der Kieler Experte wie schon so oft mit Zahlen und Fakten belegt, wie sich die Bevölkerung in Deutschland in den nächsten Jahrzehnten entwickelt und warum es Lösungsoptionen für die zukünftige Finanzierung des Gesundheitswesens geben muss. „Die Zukunft kommt, und sie kommt bald“, sagte Beske.

\section{Beitragserhöhungen und Einschränkungen}

Seinen Angaben zufolge geht die Bevölkerungszahl bis 2060 um 13 Millionen, die nachwachsende Generation um vier Millionen und die Bevölkerung im erwerbsfähigen Alter (also von 20 bis 66 Jahren) um 15 Millionen zurück. Die Babyboomer-Jahrgänge verlassen den Arbeitsmarkt ab 2016. Das bedeutet, allein die Gruppe der 67-Jährigen und älter nimmt um fünf Millionen zu. Als Rentner stehen sie dann nicht mehr als volle Beitragszahler für die Kranken- und Rentenkasse zur Verfügung. Gleichzeitig werden die Menschen immer älter und haben einen höheren Versorgungsbedarf. Beitragserhöhungen sind laut Beske die logische Folge. Seinen Rechnungen nach könnte der Beitragssatz der GKV bis 2060 bis auf 52 Prozent steigen. „Die Zuwanderung von Asylbewerbern und Flüchtlingen wird Auswirkungen haben, langfristig diese Entwicklung aber kaum verändern“, erklärte Beske und warnte: „Die Auswirkungen auf unser Sozialsystem haben schon jetzt begonnen."

Es gibt also dringenden Handlungsbedarf. In seinem Buch macht Beske konkrete und auch brisante Vorschläge. Zum Beispiel: „Die sich weiter öffnende Schere zwischen Versorgungs- bedarf in Gesundheit und Pflege bei abnehmenden finanziellen und personellen Ressourcen erfordert Einschränkungen des Leistungskatalogs der Gesetzlichen Krankenversicherung. Ziel ist die Sicherstellung der notwendigen Gesundheitsversorgung für alle." Handeln müsse die Politik - und zwar jetzt. Der Gesundheitssystem-Forscher prognostizierte etwa, dass künftig nur noch größere Krankenhäuser überleben könnten. Deshalb dürfe kein Euro mehr in ein Krankenhaus investiert werden, das keine Zukunft hat.

\section{Hausarztsystem und Bundesgesundheitsamt}

Um die medizinische Versorgung im ländlichen Raum zu gewährleisten, könne das Ziel nicht lauten: wohnortnahe Versorgung. Das wird Beske zufolge wegen eines eklatanten Mangels an Ärzten und Pflegekräften schlicht nicht möglich sein. Er setzt auf Transporte von Patienten aus ländlichen Regionen in die nächste Stadt.

Außerdem plädierte Beske für das Hausarztsystem als Grundlage für die hausärztliche Versorgung. „Das jetzige System wird durch die Finanzierung unnötiger Leistungen durch mehrere Ärzte belastet.“

Und noch ein Aufruf an die Politik: Der Experte rät die Reaktivierung des ehemaligen Bundesgesundheitsamtes. Der Gemeinsame Bundesausschuss sei zur einflussreichsten Einrichtung im Gesundheitswesen geworden. Doch bei den Entscheidungen des G-BA könne nicht ausgeschlossen werden, dass Interessen der Trägerorganisationen Entscheidungen beeinflussen. Daher solle der Gesetzgeber handeln und das Bundesgesundheitsamt neu einrichten, forderte Beske. 